\title{
Donación dirigida de sangre de Cordón Umbilical de Hermano Compatible en el Sistema de Salud Público de Chile
}

\author{
Directed sibling donor cord blood donation in the Chilean Public Health System
}

\author{
Cristián Sotomayor ${ }^{\mathrm{a}}$, Lucía Salas ${ }^{\mathrm{a}, \mathrm{b}}$, José Lattus ${ }^{\mathrm{c}}$, Alfonso T. Anguita-Compagnon ${ }^{\mathrm{d}}$, \\ Carolina Abarzúa ${ }^{\mathrm{a}}$, Paula Catalán ${ }^{\mathrm{a}}$, Julia Palma ${ }^{\mathrm{a}, \mathrm{b}}$
}

\author{
aUnidad de Trasplante de Médula Ósea, Hospital Dr. Luis Calvo Mackenna. Santiago, Chile \\ baboratorio de Procesamiento Celular, Hospital Dr. Luis Calvo Mackenna. Santiago, Chile \\ 'Hospital Dr. Luis Tisné. Santiago, Chile \\ ¿Laboratorio de Trasplante, Clínica Alemana de Santiago. Santiago, Chile
}

Recibido: 2 de octubre de 2019; Aceptado: 25 de noviembre de 2019

\section{¿Qué se sabe del tema que trata este estudio?}

El uso de sangre de cordón umbilical de recolección dirigida es de uso estándar en trasplante de progenitores hematopoyéticos. Entre 7,5 a $16,0 \%$ de las unidades recolectadas dirigidamente se utilizan. Esto permite tratar a pacientes con el mejor donante posible.

\begin{abstract}
¿Qué aporta este estudio a lo ya conocido?
Reportamos los resultados del programa público de donación dirigida de unidades sangre de cordón umbilical para trasplante de progenitores hematopoyéticos de Chile. Se logran recolecciones dentro de estándares internacionales y se han tratado 4 pacientes.
\end{abstract}

\section{Resumen}

Introducción: La sangre de cordón umbilical (SCU) como fuente para trasplante de células progenitoras hematopoyéticas (TPH) está bien establecida. Internacionalmente, menos del 10\% de los TPH de SCU corresponde a donantes hermanos compatibles. Dentro de la red del Programa Infantil Nacional de Drogas Antineoplásicas (PINDA), existe desde enero 2004 un programa de donación dirigida de SCU para TPH. Pacientes y Método: Se diseñó un estudio observacional, retrospectivo, descriptivo, se revisaron el número y características de las unidades de SCU recolectadas en el PINDA y el número, características y evolución de los pacientes trasplantados con esas unidades entre enero de 2004 y octubre de 2018. Resultados: Sesenta unidades de SCU han sido recolectadas, de ellas 55 con registro completo. La mediana de volumen de las unidades almacenadas fue 74,8 $\mathrm{ml}(30,0-170,8)$, la mediana de células nucleadas totales 7,6 x 10e8 (2,0-21,1), mediana de células CD34+1,6 x 10e6 $(0,2-11,6)$. Cuatro pacientes con leucemias de alto riesgo fueron trasplantados; mediana de seguimiento es de 8 años. Todos desarrollaron complicaciones severas post TPH, uno de ellos falleció de recaída y los tres actualmente vivos presentan un Karnofsky/Lansky 100\%. Conclusión: El programa ha permitido el trasplante de 4 pacientes que de otro modo no habrían tenido acceso a un donante. Este programa de donación dirigida puede ser considerado una primera etapa para el desarrollo de un banco público de sangre de cordón umbilical en Chile.
Palabras clave:

Trasplante de células progenitoras hematopoyéticas; Donación dirigida; Trasplante de Sangre de Cordón umbilical; Sangre de Cordón 


\begin{abstract}
Introduction: Cord blood (CB) as a source of Hematopoietic Stem Cells for Transplantation (HSCT) is well established. Worldwide, nonetheless, less than $10 \%$ of the CB HSCTs are performed with a match sibling donor. Since 2004, the Chilean National Childhood Cancer Program (PINDA) network, has established a CB directed donation program for HSCT. Patients and Method: An observational, descriptive and retrospective study was designed to assess the number and characteristics of the CB units collected in the program as well as the number, clinical characteristics and follow-up of the patients who received an HSCT from those CB units between January 2004 and October 2018. Results: Sixty CB units have been collected; 55 of them with full records and stored. The median volume collected was $74.8 \mathrm{ml}$ (30.0-170.8), the median number of total nucleated cells was $7.6 \times 10 \mathrm{e} 8$ (2.0-21.1), and the median of CD34+ cells was $1.6 \times 10 \mathrm{e} 6(0.2-11.6)$. Four high-risk leukemia patients received $\mathrm{HSCT}$, all of them developed severe complications after transplantation and one patient died due to relapse. Those patients currently alive have a 100\% Karnofsky/Lansky score. The median follow-up time was 8 years. Conclusion: The PINDA program has allowed 4 patients to be transplanted who otherwise would not have had access to a donor. This directed donation program could be seen as a model for the development of a public cord blood bank in Chile.
\end{abstract}

Keywords:

Cord Blood Stem

Cell Transplantation;

Directed Tissue

Donation; Cord Blood;

Hematopoietic Stem

Cell Transplantation

\section{Introducción}

El primer trasplante de células progenitoras hematopoyéticas (TPH) exitoso utilizando sangre de cordón umbilical (SCU) fue reportado en $1991^{1}$, actualmente el uso de SCU está establecido en niños ${ }^{2}$ y adultos ${ }^{3}$ y es frecuente en Chile y el mundo ${ }^{4}$. En 2011 se reportaba un aumento sostenido del uso de SCU pero menos del $10 \%$ correspondían a hermanos compatibles ${ }^{5}$. Desde el 2013, la utilización de SCU ha disminuido ${ }^{6}$, probablemente por las nuevas técnicas de trasplante haploidéntico. Nuevas aplicaciones de la SCU están siendo estudiadas y se prevé un nuevo aumento en su uso ${ }^{7}$.

Los resultados de TPH de SCU de hermano compatible son promisorios ${ }^{8}$. Eurocord reportó mejor sobrevida total, menor tiempo de implante y menor enfermedad del injerto contra el huésped (EICH) aguda en comparación con trasplantes de SCU no relacionados?. Además, comparando SCU y médula ósea de hermano compatible, se observa una sobrevida total similar pero menor incidencia de EICH aguda o crónica con $\mathrm{SCU}^{10}$. Ambos reportes presentan problemas metodológicos, por lo que sus conclusiones deben tomarse con cautela $^{11}$, a pesar de esto la donación dirigida de SCU para un familiar con una enfermedad potencialmente curable con TPH es altamente recomendada tanto por pediatras $^{12,13}$ como por obstetras ${ }^{14}$.

La unidad de trasplante de medula ósea (UTMO) del Hospital Dr. Luis Calvo Mackenna (HLCM) del Programa Infantil Nacional de Drogas Antineoplásicas (PINDA) inició su programa de TPH de SCU en mayo del $2003^{15}$; a la fecha se han realizado más de 140. En enero 2004 se inicia la recolección de SCU entre las madres de pacientes PINDA, con una eventual indicación de TPH. Posteriormente se amplió a pacientes sin patología oncológica atendidos en el Sistema Público de Salud. La UTMO HLCM-PINDA asumió la coordinación de los centros PINDA distribuidos por todo el país y la maternidad del Hospital Dr. Luis Tisné Brousse se constituyó como el único centro de recolección. Todas las unidades recolectadas fueron almacenadas en el Laboratorio de Trasplante de la Clínica Alemana de Santiago.

Existen pocos reportes sobre la tasa de uso de las unidades de SCU de donación dirigida y menos aún que analicen la evolución de los casos. En Chile no hay reportes de este tipo de trasplantes. El objetivo de este estudio es reportar la experiencia del programa de recolección del PINDA.

\section{Pacientes y Método}

Se diseñó un estudio retrospectivo de revisión de fichas clínicas, cuyo objetivo fue conocer el número, características y evolución de pacientes trasplantados gracias al uso de unidades de SCU recolectadas de forma dirigida. Este estudio respeta las normas éticas de la Declaración de Helsinki y contó con la aprobación por parte de la Dirección del HLCM y del Comité de Ética Científico Pediátrico del Servicio de Salud Metropolitano Oriente. Se revisaron los registros de las unidades de SCU recolectadas en el Hospital Dr. Luis Tisné Brousse para pacientes de la red PINDA entre enero 2004 y octubre 2018. Antes de acceder a las fichas, se solicitó consentimiento informado a él o a la paciente si era mayor de 18 años o a su tutor/a legal si era aun menor de 18 años. Se solicitó además asentimiento informado a la o a él paciente si era mayor de 12 años y menor de 18 años al momento del estudio. 
Se determinó el número total de unidades de sangre de cordón recolectadas, el diagnóstico de los pacientes, el hospital de derivación, el volumen, las células nucleadas totales (CNT) y las células CD34+. Desde la ficha clínica de los pacientes trasplantados se obtuvieron: diagnóstico, sexo, tratamiento y complicaciones previo al trasplante, grupo sanguíneo, acondicionamiento, edad, peso, dosis celular, implante de glóbulos rojos, glóbulos blancos y plaquetas. En el seguimiento se evaluó mielograma, quimerismo, subpoblaciones linfocitarias, inmunoglobulinas, "performance status" escala Lansky o Karnofsky según edad, complicaciones post trasplante, estado actual, tiempo de seguimiento y datos del donante: grupo sanguíneo y edad al trasplante.

\section{Resultados}

En el periodo estudiado, se recolectaron 60 unidades de SCU; 31/60 entre 2014-2018. De las 60, 3 se desecharon; una por bajo volumen extraído, una por baja celularidad y una por no cumplir con el procedimiento de extracción adecuado. De las 57 restantes, el registro estaba incompleto en 2 por lo que se excluyeron del análisis; éstas corresponden a las 2 primeras unidades extraídas. En síntesis, se analizaron 55 unidades de SCU almacenadas; sus características se resumen en la Tabla 1 .
De las 5 unidades de SCU almacenadas para pacientes con patología no oncológica, ninguna se ha utilizado. De las unidades de SCU almacenadas para pacientes con patología oncológica, 4 se han utilizado en 4 pacientes. Esto es un 6,7\% del total de las 60 unidades recolectadas, 7,0\% de las 57 almacenadas y 8,0\% de las 50 almacenadas por patología oncológica.

Previo al trasplante, dos de los cuatro pacientes trasplantados fueron tratados en el HLCM de Santiago, uno en el Hospital Dr. Sótero del Río de Santiago y uno en el Hospital Dr. Gustavo Fricke de Viña del Mar. La evolución de los pacientes pre trasplante se resume en la Tabla 2.

La diferencia de edad entre el donante y el receptor, fluctuó entre 1,5 y 14,9 años. En los adolescentes trasplantados, pacientes 2 y 3 , las dosis celulares del cordón eran bajas; el paciente 3 recibió además médula ósea para aumentar la dosis celular. La Tabla 3 resume las características de pacientes y donantes al momento del trasplante.

Los cuatro pacientes recibieron las profilaxis estándar: Neomicina, Aciclovir, Fluconazol, Heparina y Cotrimoxazol después del implante. La inmunosupresión fue en base a Ciclosporina, en el paciente 4 por toxicidad se cambió a Tacrolimus. Todos implantaron, sin embargo el paciente 4 recae y fallece a 4 meses del trasplante. Los tres pacientes que no han recaído, presentaron múltiples complicaciones, algunas de ellas raras como síndrome de stiff person o síndrome de persona

Tabla 1. Características de las unidades de sangre de cordón almacenadas

\begin{tabular}{|c|c|c|c|c|}
\hline \multirow[t]{2}{*}{ Características } & \multicolumn{3}{|c|}{ Años } & \multirow[t]{2}{*}{ Total } \\
\hline & $2004-2008$ & $2009-2013$ & $2014-2018$ & \\
\hline Número & $6^{*}$ & 18 & 31 & 55 \\
\hline \multicolumn{5}{|l|}{ Recolección por diagnóstico } \\
\hline No oncológico & 2 & 2 & 1 & $5(9 \%)$ \\
\hline Oncológico & 4 & 16 & 30 & $50 \quad(91 \%)$ \\
\hline \multicolumn{5}{|l|}{ Hospital de derivación } \\
\hline RM & 3 & 14 & 27 & $44 \quad(80 \%)$ \\
\hline No RM & 1 & 1 & 4 & $6(11 \%)$ \\
\hline No dato & 2 & 3 & 0 & $5(9 \%)$ \\
\hline Mediana de volumen (ml) & $\begin{array}{c}70,0 * * \\
\text { (rango 60,5-91,0) }\end{array}$ & $\begin{array}{c}73,7 \\
\text { (rango } 31,5-125,3 \text { ) }\end{array}$ & $\begin{array}{c}80,6 \\
\text { (rango 30,0-170,8) }\end{array}$ & $\begin{array}{c}74,8 \\
\text { (rango } 30,0-170,8)\end{array}$ \\
\hline $\begin{array}{l}\text { Mediana de CNT } \\
\left(x 10^{8}\right)\end{array}$ & $\begin{array}{c}6,5 \\
\text { (rango 5,3-14,1) }\end{array}$ & $\begin{array}{c}7,8 \\
\text { (rango } 2,7-16,5 \text { ) }\end{array}$ & $\begin{array}{c}7,9 \\
\text { (rango 0,2-21,2) }\end{array}$ & $\begin{array}{c}7,6 \\
\text { (rango } 2,0-21,1 \text { ) }\end{array}$ \\
\hline $\begin{array}{l}\text { Mediana de CD34 } \\
\left(\times 10^{6}\right)\end{array}$ & $\begin{array}{c}1,0 \\
\text { (rango } 0,5-8,6)\end{array}$ & $\begin{array}{c}1,6 \\
\text { (rango } 0,4-3,7 \text { ) }\end{array}$ & $\begin{array}{c}1,6 \\
\text { (rango } 0,2-11,2 \text { ) }\end{array}$ & $\begin{array}{c}1,6 \\
\text { (rango } 0,2-11,2 \text { ) }\end{array}$ \\
\hline
\end{tabular}

RM: hospital de la región Metropolitana, No RM: hospital fuera de la región Metropolitana, CNT: Células nucleadas totales, CD34: Células CD34 +. * se excluyen 2 unidades por registro de datos insuficiente. **datos disponibles sólo en 5 de las 6 unidades. 
Tabla 2. Diagnóstico y evolución pre trasplante de los pacientes

\begin{tabular}{|c|c|c|c|c|}
\hline & Sexo & DGCO & Tratamiento & Complicaciones pre TPH \\
\hline 1 & $\mathrm{~F}$ & LLA 2RC & $\begin{array}{l}\text { LLA PINDA } 2002 \\
\text { IDA-FLAG } \\
\text { Consolidación } \\
\text { TG y MTX }\end{array}$ & $\begin{array}{l}\text { Sinusitis a repetición } \\
\text { SDA Clostridium difficile } \\
\text { Neumonia Pneumocystis jirovecii con VMI } \\
\text { Candidemia Candida tropicalis } \\
\text { Hemorragia digestiva alta }\end{array}$ \\
\hline 2 & M & $\begin{array}{l}\text { LLA Phi(+) } \\
\quad 1 \text { RC }\end{array}$ & ESPHALL & $\begin{array}{l}\text { Toxocariasis } \\
\text { Serología Toxoplasma positiva } \\
\text { PCR BCR/ABL cualitativa + pre TPH }\end{array}$ \\
\hline 3 & M & $\begin{array}{l}\text { LMC } \\
1 \text { FC }\end{array}$ & $\begin{array}{l}\text { Hidroxiurea } \\
\text { Imatinib }\end{array}$ & $\begin{array}{l}\text { Gastropatía por prolapso } \\
\text { Parestesias secundarias a Imatinib } \\
\text { Sin remisión molecular estable }\end{array}$ \\
\hline 4 & M & LMMJ en actividad & $\begin{array}{c}\text { Vall d’Hebron } \\
\text { LMA PINDA } 2006\end{array}$ & $\begin{array}{l}\text { Dermatitis atópica } \\
\text { Toxocariasis } \\
\text { Fimosis } \\
\text { SBOR } \\
\text { Reacción adversa a transfusiones }\end{array}$ \\
\hline
\end{tabular}

DGCO: diagnóstico que motiva el trasplante, TPH: trasplante de precursores hematopoyéticos F: femenino, M: masculino, LLA 2RC: leucemia linfoblástica aguda en segunda remisión completa, LLA PINDA 2002: protocolo de tratamiento de leucemia linfoblástica aguda del PINDA versión 2002. IDA-FLAG: tratamiento con idarrubicina, fludarabina, citarabina filgrastim. Consolidación: tratamiento con etopósido y citarabina. TG y MTX: tratamiento de mantención con metotrexato y tioguanina, SDA: síndrome diarreico agudo, VMl: ventilación mecánica invasiva, 1RC: primera remisión completa, Ph (+): cromosoma filadelfia positivo, ESPhALL: protocolo de tratamiento de la leucemia linfoblástica aguda con cromosoma filadelfia positivo en el grupo PINDA. LMC 1FC: leucemia mieloide crónica en primera fase crónica, LMMJ: leucemia mielomonocítica juvenil, Vall d’Hebron: protocolo de tratamiento ${ }^{16}$, LMA PINDA 2006: protocolo de tratamiento de la leucemia mieloide aguda del PINDA versión 2006. SBOR: síndrome bronquial obstructivo recurrente.

Tabla 3. Características clínicas de los pacientes y de los donantes

\begin{tabular}{|c|c|c|c|c|c|c|c|c|}
\hline & \multicolumn{4}{|c|}{ Paciente } & \multicolumn{4}{|c|}{ Donante } \\
\hline & Sexo & Edad y peso* & CMV & Grupo & CNT & Edad** $^{*}$ & Grupo & Sexo \\
\hline 1 & $\mathrm{~F}$ & $\begin{array}{l}4,7 \text { años } \\
15,0 \mathrm{Kg}\end{array}$ & $(+)$ & A Rh+ & $3,5 \times 10^{7} / \mathrm{Kg}$ & 8 meses & $0 \mathrm{Rh}+$ & $\mathrm{F}$ \\
\hline 2 & M & $\begin{array}{l}14,0 \text { años } \\
53,0 \mathrm{Kg}\end{array}$ & $(+)$ & $0 \mathrm{Rh}+$ & $1,9 \times 10^{7} / \mathrm{Kg}$ & 3 meses & $0 \mathrm{Rh}+$ & M \\
\hline 3 & M & $\begin{array}{l}17,9 \text { años } \\
62,5 \mathrm{Kg}\end{array}$ & $(-)$ & $0 \mathrm{Rh}+$ & $\begin{array}{l}\text { SCU: } 0,2 \times 10^{7} / \mathrm{Kg} \\
\text { MO: } 11,8 \times 10^{7} / \mathrm{Kg}\end{array}$ & 3 años & $0 \mathrm{Rh}+$ & $\mathrm{F}$ \\
\hline 4 & M & $\begin{array}{c}2,0 \text { años } \\
13,7 \mathrm{Kg}\end{array}$ & $(-)$ & $0 \mathrm{Rh}+$ & $4,3 \times 10^{7} / \mathrm{kg}$ & 6 meses & $0 \mathrm{Rh}+$ & $\mathrm{F}$ \\
\hline
\end{tabular}

Peso*: peso al momento del trasplante, CMV: serología citomegalovirus, CNT: células nucleadas totales infundidas, Edad**: edad al momento del trasplante, F: femenino, M: masculino, SCU: sangre de cordón umbilical, MO: médula ósea.

rígida (paciente 3) o graves como cáncer secundario de tiroides (paciente 1). A pesar de lo anterior, están actualmente en buenas condiciones con Lansky/Karnofsky $100 \%$ con una mediana de seguimiento de 8 años. Los tres han logrado una inmunorreconstitución adecuada y han completado la vacunación post trasplante. Los pacientes 2 y 3 cursan estudios superiores y la paciente 1 está completando la educación media. Ninguno de los pacientes reside en la Región Metropolitana. La evolución de los pacientes se resume en la Tabla 4.

\section{Discusión}

El donante familiar idéntico es considerado como el donante ideal dentro de los TPH alogénicos ${ }^{17}$ y se prefiere por sobre otros donantes ${ }^{18}$.

Nuestro datos muestran cómo la recolección de cordones ha aumentado quinquenio a quinquenio. Dado que el PINDA es un grupo que trata cáncer, el 91\% de las unidades de SCU almacenadas han sido por patología oncológica; de ellas se han utilizado el $8 \%$. 
Tabla 4. Trasplante y evolución post trasplante de los pacientes

\begin{tabular}{|c|c|c|c|c|c|}
\hline & Acondicionamiento & Implante & $\begin{array}{l}\text { Complicaciones Precoces } \\
<100 \text { días post TPH }\end{array}$ & $\begin{array}{l}\text { Complicaciones } \\
\text { tardías }\end{array}$ & Seguimiento \\
\hline 1 & $\begin{array}{l}\text { VP16 } 30 \mathrm{mg} / \mathrm{kg} \\
\text { Cy } 200 \mathrm{mg} / \mathrm{kg} \\
\text { ICT } 12 \mathrm{~Gy}\end{array}$ & $\begin{array}{l}G B+21 \\
G R+20 \\
P L T+39\end{array}$ & $\begin{array}{c}\text { Bacteriemia por S. mutans, } \\
\text { Pansinusitis }\end{array}$ & $\begin{array}{l}\text { Neumopatía VRS } \\
\text { Hipotiroidismo } \\
\text { Cáncer de Tiroides }\end{array}$ & $\begin{array}{l}\text { 11,1 años post TPH } \\
\text { Quimerismo } 100 \% \\
\text { Lansky } 100 \% \\
\text { Sin EICH } \\
\text { RC de ambos cánceres } \\
\text { IR a } 1 \text { año post TPH }\end{array}$ \\
\hline 2 & $\begin{array}{l}\text { VP16 } 30 \mathrm{mg} / \mathrm{kg} \\
\text { Cy } 120 \mathrm{mg} / \mathrm{kg} \\
\text { ICT } 12 \mathrm{~Gy}\end{array}$ & $\begin{array}{l}G B+26 \\
G R+31 \\
P L T+45\end{array}$ & $\begin{array}{l}\text { Convulsión focal } \\
\text { Shock séptico K. oxytoca } \\
\text { EICH Ag Grado } 2 \\
\text { CMV } 2 \text { veces }\end{array}$ & $\begin{array}{l}\text { EICH Cr cutánea } \\
\text { CMV 3era vez }\end{array}$ & $\begin{array}{c}\text { 9,3 años post TPH } \\
\text { Quimerismo } 100 \% \\
\text { Lansky } 100 \% \\
\text { Sin EICH } \\
\text { RC } \\
\text { IR a } 1 \text { año post TPH }\end{array}$ \\
\hline 3 & $\begin{array}{l}\mathrm{Bu} 16 \mathrm{mg} / \mathrm{kg} \\
\mathrm{Cy} 120 \mathrm{mg} / \mathrm{kg}\end{array}$ & $\begin{array}{l}G B+13 \\
G R+15 \\
P L T+29\end{array}$ & $\begin{array}{l}\text { Infección CVC S. epidermidis } \\
\text { Sd hemofagocítico } \\
\text { Insuficiencia renal aguda } \\
\text { Sd deprivación de morfina }\end{array}$ & $\begin{array}{l}\text { EICH Cr pulmonar } \\
\text { Sd. persona rígida } \\
\text { Litiasis renal }\end{array}$ & $\begin{array}{c}6,6 \text { años post TPH } \\
\text { Quimerismo } 100 \% \\
\text { Karnofsky } 100 \% \\
\text { Sin EICH } \\
\text { RC } \\
\text { IR a } 1 \text { año post TPH }\end{array}$ \\
\hline 4 & $\begin{array}{l}\text { Bu } 24 \mathrm{mg} / \mathrm{kg} \\
\text { Cy } 120 \mathrm{mg} / \mathrm{kg} \\
\text { Mel } 145 \mathrm{mg} / \mathrm{m}^{2}\end{array}$ & $\begin{array}{l}\mathrm{GB}+23 \\
\mathrm{GR}+33 \\
\mathrm{PLT} N / A\end{array}$ & $\begin{array}{c}\text { EICH Ag Grado } 2 \\
\text { SIRS responde a esteroides } \\
\text { MAT por Csa } \\
\text { Cistitis Hemorrágica por BK } \\
\text { VHH6 en MO } \\
\text { Recaída post TPH }(+73)\end{array}$ & N/A & $\begin{array}{c}\text { Fallecido } 4 \text { meses } \\
\text { post TPH }\end{array}$ \\
\hline
\end{tabular}

VP16: etopósido, Cy ciclofosfamida, ICT: irradiación corporal total, GB glóbulos blancos, GR glóbulos rojos, PLT: plaquetas, VRS: virus respiratorio sincicial, EICH: enfermedad del injerto contra el hospedero, RC: en remisión completa, IR: inmunorreconstitución, Ag: aguda, Cr: crónica, CMV infección/reactivación citomegalovirus, Bu: busulfan oral, CVC: catéter venoso central, Sd: síndrome, Mel: melfalán, SIRS: síndrome de respuesta inflamatoria sistémica, MAT: microangiopatía trombótica, CSA: ciclosporina, BK: virus BK, VHH6: virus Herpes humano 6, MO: médula ósea, TPH: trasplante de precursores hematopoyéticos.

En Grecia se reportan trasplantes en el 2\% de las unidades recolectadas por patología maligna ${ }^{19}$ y el $16 \%$ de aquellas por hemoglobinopatías ${ }^{10}$. En Italia, un programa de más de 20 años trasplanta el 7,35\% unidades en general ${ }^{21}$ pero sólo $3,4 \%$ en leucemia ${ }^{22}$.

En nuestro programa la mediana de volumen y CNT es mayor quinquenio a quinquenio, reflejando la mayor experiencia de los equipos; se logran volúmenes y CNT similares a los reportados pero el contenido de $\mathrm{CD} 34+$, es menor ${ }^{19-21,23}$. El uso simultáneo médula ósea y SCU de un hermano compatible parece ser beneficioso ${ }^{24}$ y se realizó en el paciente 3; por lo que una unidad aunque tenga recuentos bajos de CD34, se debe guardar.

En nuestros pacientes, la diferencia de edad con los donantes fue tal que habría sido imposible la donación de médula ósea. Los pacientes son sólo 4 pero reflejan bien las potenciales complicaciones complejas de los trasplantados, algunas de ellas con manifestaciones a muy largo plazo. Son necesarios seguimientos prolongados y una adecuada transición a la medicina de adultos de los sobrevivientes de cáncer infantil. Este es un desafío pendiente en Chile.

El programa de donación dirigida de hermanos del PINDA, representa un desafío para el Sistema de Salud Público chileno, requiere la coordinación en red de múltiples equipos para su éxito; al menos $11 \%$ de las unidades almacenadas provienen de derivaciones fuera de la región Metropolitana. La coordinación ha sido posible gracias a más de 20 años de funcionamiento del comité de TPH del PINDA. La donación dirigida de SCU a hermanos enfermos está fuertemente aconsejada y se debería promover su expansión en el futuro ${ }^{5}$. El programa de recolección y almacenamiento, puede servir de modelo para el desarrollo de un banco de sangre de cordón umbilical en el Sistema Público de Salud.

\section{Responsabilidades Éticas}

Protección de personas y animales: Los autores declaran que los procedimientos seguidos se conformaron 
a las normas éticas del comité de experimentación humana responsable y de acuerdo con la Asociación Médica Mundial y la Declaración de Helsinki.

Confidencialidad de los datos: Los autores declaran que han seguido los protocolos de su centro de trabajo sobre la publicación de datos de pacientes.

Derecho a la privacidad y consentimiento informado: Los autores han obtenido el consentimiento in- formado de los pacientes y/o sujetos referidos en el artículo. Este documento obra en poder del autor de correspondencia.

\section{Conflicto de intereses}

Los autores reconocen como conflicto de interés que CS, JL, CA, PC y JP fueron médicos tratantes directos de los pacientes involucrados en el presente estudio.

\section{Referencias}

1. Gluckman E, Broxmeyer HA, Auerbach $\mathrm{AD}$, et al. Hematopoietic reconstitution in a patient with Fanconi's anemia by means of umbilical-cord blood from an HLA-identical sibling. N Engl J Med. 1989;321:1174-8.

2. Peters C, Cornish JM, Parikh SH, Kurtzberg J. Stem cell source and outcome after hematopoietic stem cell transplantation (HSCT) in children and adolescents with acute leukemia. Pediatr Clin North Am. 2010;57:27-46.

3. Brunstein CG, Laughlin MJ. Extending cord blood transplant to adults: dealing with problems and results overall. Semin Hematol. 2010;47:86-96.

4. Barriga F, Ramírez P, Wietstruck A, Rojas N. Hematopoietic stem cell transplantation: clinical use and perspectives. Biol. Res. [online]. 2012;45:307-316.

5. Gluckman E, Ruggeri A, Rocha V, et al. Eurocord, Netcord, World Marrow Donor Association and National Marrow Donor Program. Familydirected umbilical cord blood banking. Haematologica. 2011;96:1700-7.

6. Dessels C, Alessandrini M, Pepper MS, Factors Influencing the Umbilical Cord Blood Stem Cell Industry: An Evolving Treatment Landscape. Stem Cells Transl Med. 2018;7:643-650.

7. Mayani H, Wagner JE, Broxmeyer HE. Cord blood research, banking, and transplantation: achievements, challenges, and perspectives. Bone Marrow Transplant. 2019 May 14. doi: 10.1038/ s41409-019-0546-9.

8. Wagner JE, Kernan NA, Steinbuch M, Broxmeyer HE, Gluckman E. Allogeneic sibling umbilical-cordblood transplantation in children with malignant and non-malignant disease. . Lancet. 1995;346:214-9.

9. Gluckman E, Rocha V, Boyer-Chammard A, et al. Outcome of cord-blood transplantation from related and unrelated donors. Eurocord Transplant Group and the European Blood and Marrow Transplantation Group. N Engl J Med. 1997;337:373-81

10. Rocha V, Wagner JE Jr, Sobocinski KA, Klein JP, Zhang MJ, Horowitz MM, Gluckman E. Graft-versus-host disease in children who have received a cordblood or bone marrow transplant from an HLA-identical sibling. Eurocord and International Bone Marrow Transplant Registry Working Committee on Alternative Donor and Stem Cell Sources. N Engl J Med. 2000;342:1846-54.

11. Schmidt A, Platz A, Rutt C, Ehninger G. Making the Case for Private Cord Blood Banking: Mission Failed! Stem Cell Rev and Rep 2010;6:234-6

12. Shearer WT, Lubin BH, Cairo MS, Notarangelo LD; Section on Hematology/ Oncology; Section on Allergy and Immunology. Cord Blood Banking for Potential Future Transplantation. Pediatrics. 2017;140(5):e20172695. doi:10.1542/peds.2017-2695.

13. Thornley I, Eapen M, Sung L, Lee SJ, Davies SM, Joffe S. Private cord blood banking: experiences and views of pediatric hematopoietic cell transplantation physicians. Pediatrics. 2009;123:1011-7.

14. Armson BA. Umbilical cord blood banking: implications for perinatal care providers. J Obstet Gynaecol Can. 2005;27:263-90.

15. Palma J, Mosso C, Paris C, et al. Establishment of a pediatric HSCT program in a public hospital in Chile. Pediatr Blood Cancer. 2006;46:803-10.

16. Díaz de Heredia C, Ortega JJ, Coll MT, Bastida P, Olivé T. Results of intensive chemotherapy in children with juvenile chronic myelomonocytic leukemia: a pilot study. Med Pediatr Oncol. 1998;31:51620.

17. Ljungman P, Bregni M, Brune $M$, et al. Allogeneic and autologous transplantation for haematological diseases, solid tumour and immune disorders: current practice in Europe 2009. Bone Marrow Transplant. 2010;45:219-34.

18. Hough R, Cooper N, Veys P. Allogeneic haemopoietic stem cell transplantation in children: what alternative donor should we choose when no matched sibling is available? Br J Haematol. 2009; 147:593613.

19. Goussetis E, Peristeri I, Kitra V, et al. Low usage rate of banked sibling cord blood units in hematopoietic stem cell transplantation for children with hematological malignancies: implications for directed cord blood banking policies. Blood Cells Mol Dis. 2011;46:177-81.

20. Goussetis E, Petrakou E, Theodosaki $\mathrm{M}$, et al. Directed sibling donor cord blood banking for children with betathalassemia major in Greece: usage rate and outcome of transplantation for HLA-matched units. Blood Cells Mol Dis. 2010;44:107-10.

21. Screnci M, Murgi E, Valle V, et al. Sibling cord blood donor program for hematopoietic cell transplantation: the 20-year experience in the Rome Cord Blood Bank. Blood Cells Mol Dis. 2016;57:71-3.

22. Screnci M, Murgi E, Tamburini A, et al. Family directed umbilical cord blood banking for acute leukemia: usage rate in hematopoietic stem cell transplantation. Stem Cell Rev. 2015;11:275-9.

23. Smythe J, Armitage S, McDonald D et al. Direct sibling cord blood banking for transplantation: the 10-year experience in the national blood service in England. Stem Cells 2007;25:2087-93.

24. Soni S, Boulad F, Cowan MJ, et al. Combined Umbilical Cord Blood and Bone Marrow from HLA-Identical Sibling Donors for Hematopoietic Stem Cell Transplantation in Children with Hemoglobinopathies Pediatr Blood Cancer 2014;61:1690-94. 